\title{
Complete genome sequence of the entomopathogenic and metabolically versatile soil bacterium Pseudomonas entomophila
}

\author{
Nicolas Vodovar ${ }^{1}$, David Vallenet ${ }^{2}$, Stéphane Cruveiller ${ }^{2}, Z_{\text {Zoé }}$ Rouy $^{2}$, Valérie Barbe ${ }^{2}$, Carlos Acosta ${ }^{1}$, \\ Laurence Cattolico $^{2}$, Claire Jubin ${ }^{2}$, Aurélie Lajus ${ }^{2}$, Béatrice Segurens ${ }^{2}$, Benoit Vacherie ${ }^{2}$, Patrick Wincker ${ }^{2}$, \\ Jean Weissenbach ${ }^{2}$, Bruno Lemaitre ${ }^{1}$, Claudine Médigue $^{2} \&$ Frédéric Boccard $^{1}$ \\ Pseudomonas entomophila is an entomopathogenic bacterium that, upon ingestion, kills Drosophila melanogaster as well as \\ insects from different orders. The complete sequence of the 5.9-Mb genome was determined and compared to the sequenced \\ genomes of four Pseudomonas species. $P$. entomophila possesses most of the catabolic genes of the closely related strain \\ P. putida KT2440, revealing its metabolically versatile properties and its soil lifestyle. Several features that probably contribute \\ to its entomopathogenic properties were disclosed. Unexpectedly for an animal pathogen, $P$. entomophila is devoid of a type III \\ secretion system and associated toxins but rather relies on a number of potential virulence factors such as insecticidal toxins, \\ proteases, putative hemolysins, hydrogen cyanide and novel secondary metabolites to infect and kill insects. Genome-wide \\ random mutagenesis revealed the major role of the two-component system GacS/GacA that regulates most of the potential \\ virulence factors identified.
}

Pseudomonas spp. are ubiquitous Gram-negative bacteria that colonize and survive in numerous ecological niches including soil, water and plant surfaces. This versatility is reflected by the sizes of their genomes, which contain large sets of genes involved in carbon source utilization and adaptation. In 2001, we isolated a bacterial strain closely related to the saprophytic soil bacterium Pseudomonas putida, Pseudomonas entomophila, which triggers a systemic immune response in D. melanogaster after ingestion ${ }^{1}$. P. entomophila is highly pathogenic for both $D$. melanogaster larvae and adults. Its persistence in larvae leads to a massive destruction of gut cells ${ }^{1}$.

Entomopathogenic bacteria such as the Gram-negative bacteria Photorhabdus luminescens, Xenorhabdus nematophilus, Yersinia pestis, Serratia marcescens and Serratia entomophila and the Grampositive bacterium Bacillus thuringiensis have developed different strategies to interact with and kill insects ${ }^{2}$. Some gene products derived from these bacteria as well as the bacteria themselves, have been used to generate biopesticides ${ }^{3}$. The ability of $P$. entomophila to orally infect and kill larvae of insect species belonging to different orders makes it a promising model for the study of host-pathogen interactions and for the development of biocontrol agents against insect pests. To unravel features contributing to $P$. entomophila's entomopathogenic properties, we have determined its complete genome sequence and performed a genome-wide screen for mutants affected in their ability to trigger an immune response and lethality in D. melanogaster.

\section{RESULTS}

Genome features and comparative genomics

The P. entomophila genome is composed of a single circular chromosome of 5,888,780 base pairs (Fig. 1). Among 5,169 coding sequences identified, 3,466 genes (67\%) have been assigned a predicted function (Table 1). The P. entomophila genome is smaller than the six other Pseudomonas genomes that have been published (Table 1): the human opportunistic pathogen $P$. aeruginosa PAO1 (ref. 4), the three $P$. syringae pathovars ${ }^{5-7}$, the plant commensal $P$. fluorescens Pf-5 (ref. 8) and the saprophytic soil bacterium P. putida KT2440 (ref. 9).

GC skew analysis and the predicted location of the origin of replication oriC near $d n a A$ and of the chromosome dimer resolution dif site in PSEEN2780 revealed the presence of two replichores of similar size, contrary to the unbalanced replichores found in the genomes of $P$. putida KT2440 (ref. 10) and P. aeruginosa PAO1 (ref. 4) (see Supplementary Fig. 1 online). BLAST comparisons of genomes from the five Pseudomonas representative species identified a set of 2,065 genes that constitutes the Pseudomonas core genome. Based on this analysis, we identified 1,002 genes unique to the P. entomophila genome. We found that, consistent with the close relatedness between P. entomophila and P. putida ${ }^{1}, 70.2 \%$ of $P$. entomophila genes $(3,630)$ have orthologs in the P. putida genome, of which more than $96 \%$ are found in synteny (see Supplementary Table 1 online). The smaller size of the $P$. entomophila genome compared to that of other Pseudomonas does not seem to originate from reductive evolution.

${ }^{1}$ Centre de Génétique Moléculaire, Centre National de la Recherche Scientifique, 91198 Gif-sur-Yvette, France. ${ }^{2}$ Genoscope, Centre National de Séquençage and CNRSUMR8030, 2 rue Gaston Crémieux, 91057 Evry Cedex, France. Correspondence should be addressed to F.B. (boccard@cgm.cnrs-gif.fr). 


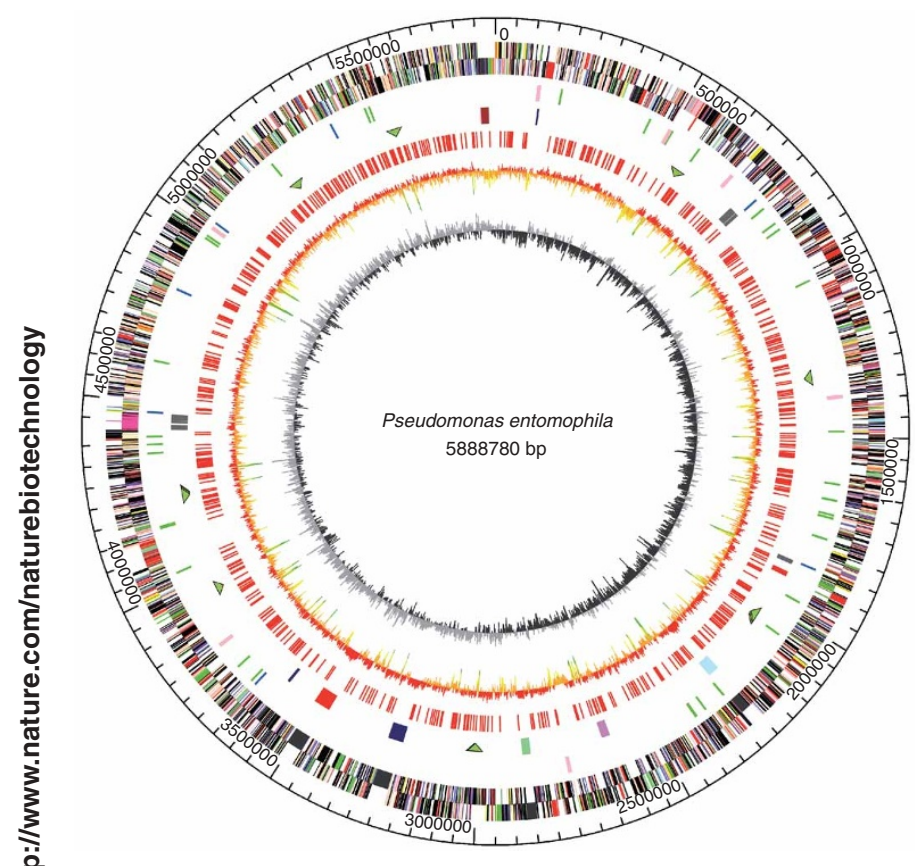

Indeed the 50 genes of P. entomophila present in other Pseudomonas but absent from $P$. putida belong to functional classes as diverse as the 34 genes of P. putida present in other Pseudomonas but absent from P. entomophila. Furthermore, comparison of gene contents in P. entomophila and P. putida indicates that the higher number of species-specific genes in $P$. putida $(1,774$ versus 1,539$)$ largely results from the presence of a higher number of paralogous genes (Fig. 2 and Supplementary Table 2 online). Comparison of the chromosome structures of P. entomophila and P. putida KT2440 and scatter plot analysis of syntenic regions of the two strains revealed frequent genetic inversions that reverse the genomic sequence symmetrically across (2) oriC as observed in other bacterial genera ${ }^{11}$ (Fig. 2 and Supplementary Fig. 2 online). The same rearrangement profile was observed when comparing the P. entomophila genome with those of other Pseudomonas spp., even though the levels of orthology and of synteny were lower (see Supplementary Table 1 and Supplementary Fig. 2 online). A search for repetitive extragenic palindromic sequences (REPs) identified 943 REPs similar to those found in the genomes of $P$. putida KT2440 (ref. 12) and P. fluorescens Pf-5 (ref. 8). The genome of P. entomophila has been remodeled by genetic mobile elements and bacteriophage insertions considerably less than the genomes of other environmental pseudomonads such as P. putida KT2440 and P. syringae pv. tomato DC3000 (Fig. 1). Particularly notable are three clustered prophages related to FluMu phage, a pyocin-like phage and a lambdoid phage; they are inserted between recA and mutS, as observed for FluMu phage in $P$. fluorescens Pf-5 genome. Also of particular interest are two putative prophages inserted in genes encoding 4.5S RNA and tmRNA, respectively. The genome of $P$. entomophila contains only nine genes encoding
Figure 1 Circular representation of the $P$. entomophila genome. The outer scale indicates coordinates in base pairs (bp). Circles 1 and 2 (from outside to inside) show predicted coding regions transcribed clockwise and counterclockwise, respectively. Coding sequences are color coded by role categories: salmon, amino acid biosynthesis; light blue, biosynthesis of cofactors, prosthetic groups and carriers; light green, cell envelope; red, cellular processes; brown, central intermediary metabolism; yellow, DNA metabolism; green, energy metabolism; purple, fatty acid and phospholipid metabolism; violet, mobile and extrachromosomal element functions; pink, protein synthesis and fate; orange, purines, pyrimidines, nucleosides and nucleotides; navy blue, regulatory functions and signal transduction; lime green, secondary metabolite biosynthesis; gray, transcription; teal, transport and binding proteins; black, unknown function and hypothetical proteins. Circle 3 shows rRNA genes in salmon, tRNA genes in green and miscellaneous RNA genes in blue. Circle 4 shows transposase genes, putative prophages and gene clusters encoding secondary metabolites coded by colored symbols as follows: green arrowheads, transposases; gray, putative prophages; red, pyoverdine synthesis; light blue, cluster involved in lipopeptide II biosynthesis; violet, acinetobactin-like siderophore synthesis; light green, cluster involved in lipopeptide III biosynthesis; navy blue, cluster and isolated genes involved in lipopeptide I biosynthesis; pink, hydrogen cyanide production; brown, polyketide synthesis. Circle 5 shows the distribution of REPs. These repeats are scattered all over the genome and were found either as single elements, in paired elements or in clusters of up to six elements in alternating orientation. Circle 6 shows $G+C$ in relation to the mean $\mathrm{G}+\mathrm{C}$ in a 1,000-bp window. Circle 7 shows $\mathrm{GC}$ skew in a 1,000-bp window.

transposase-like proteins including three that are remnant or inactive. Unlike the genomes of $P$. putida KT2440 and P. syringae pv. tomato DC3000, the genome of $P$. entomophila is devoid of type II introns.

\section{Toxins against insects}

We used several criteria to uncover genes that may contribute to the entomopathogenic properties of $P$. entomophila: specificity to the $P$. entomophila genome, localization within genomic islands that suggest recent lateral acquisitions (based on break of the synteny, GC content and absence of REPs) and similarity to genes associated with virulence in other systems (Table 2).

Particularly striking are three genes absent from other Pseudomonas genomes that encode proteins related to insecticidal toxin complexes that have been found only in entomopathogenic enterobacteria such as Photorhabdus luminescens, Serratia entomophila, Xenorhabdus nematophilus or in Yersinia spp. ${ }^{13,14}$. Three basic types of genetic

Table 1 General features of genomes of representative Pseudomonas species

\begin{tabular}{lccccr}
\hline General features & $P e$ & $P p^{\mathrm{a}}$ & $P f^{\mathrm{a}}$ & $P a^{\mathrm{a}}$ & $P s t^{\mathrm{a}}$ \\
\hline Size (Mb) & 5.9 & 6.2 & 7.1 & 6.3 & 6.4 \\
GC (\%) & 64.2 & 61.6 & 63.3 & 66.6 & 58.4 \\
Nb CDS & 5169 & 5420 & 6144 & 5570 & 5615 \\
Coding (\%) & 89.1 & 87.7 & 88.8 & 89 & 86.8 \\
rRNA operon & 7 & 7 & 5 & 4 & 5 \\
tRNA & 78 & 74 & 71 & 63 & 63 \\
& & & & & \\
Protein with predicted function (\%) & 67.1 & 65.8 & 62.2 & 54.2 & 61.0 \\
Proteins without predicted function & & & & & \\
$\quad$ Conserved hypothetical proteins (\%) & 25.3 & 19.1 & 32.5 & 13.8 & 17.0 \\
$\quad$ Hypothetical proteins (\%) & 7.5 & 15.1 & 5.3 & 31.9 & 22.0 \\
\hline
\end{tabular}

The distributions of ORFs for the published chromosomes are derived from the original annotation. These numbers, particularly those of hypothetical and conserved hypothetical proteins, may be different from numbers obtained with updated BLAST searches and annotations. Features of the genomes of $P$. syringae pv. syringae $\mathrm{B} 728 \mathrm{a}(6.1 \mathrm{Mb})$ and $P$. syringae pv. phaesolicola $1448 \mathrm{~A}$ $(5.9 \mathrm{Mb})$ are not indicated. CDS, coding sequences; $P$ e, $P$. entomophila; $P$ a, Pseudomonas aeruginosa; Pp, Pseudomonas putida Pf, Pseudomonas fluorescens Pf-5; Pst, Pseudomonas syringae pv. tomato DC3000. 
a

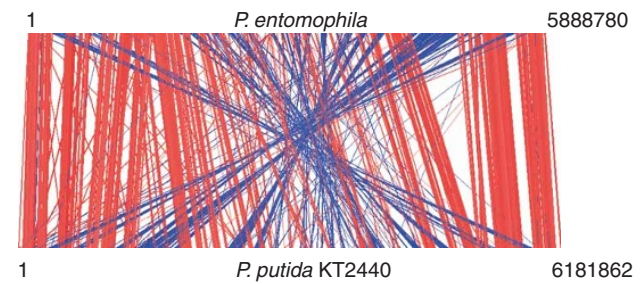

b

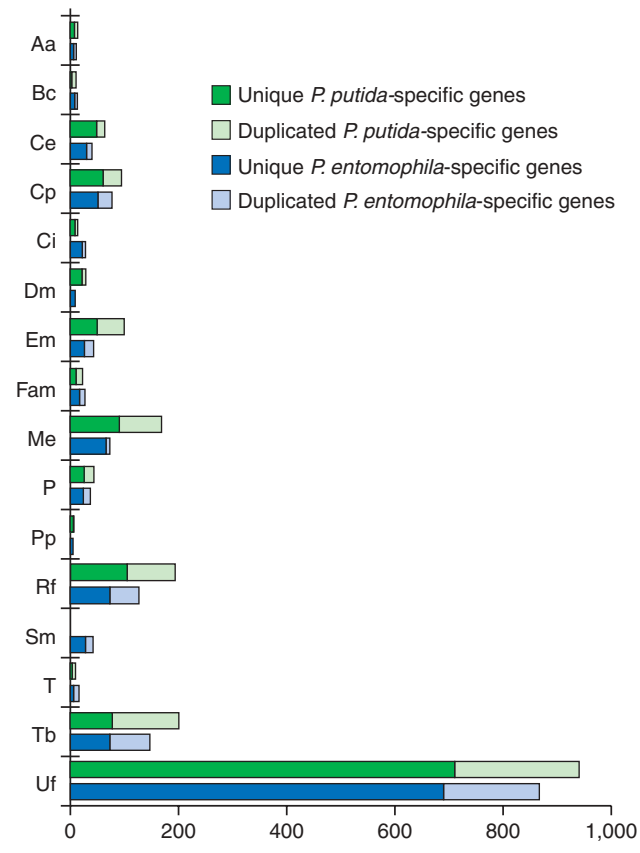

elements encode insecticidal toxin complexes: $t c d A-, t c d B$ - and $t c c C$ like genes. The $P$. entomophila genome encodes three TccC-type insecticidal toxins (PSEEN2485, PSEEN2697, PSEEN2788) (see Supplementary Fig. 3 online). In addition to these three insecticidal (2) toxins, the P. entomophila genome, like that of P. syringae, encodes proteins more distantly related to TccC-type toxins (PSEEN701 and PSEEN702) and to TcdB-type toxins (PSEEN1172). The three $P$. entomophila insecticidal toxins likely play a major role in the pathogenicity of $P$. entomophila as $\mathrm{TccC}$ and $\mathrm{TcdB}$ proteins have been shown to have entomocidal activity ${ }^{15,16}$, even though the molecular mechanisms remain to be characterized. These findings highlight the efficient spreading of toxin-complex gene homologs in insect-interacting soil bacteria belonging to different genera.

Bacterial hemolysins are exotoxins that attack blood cell membranes and cause cell rupture by poorly defined mechanisms ${ }^{17}$. Contrary to the other Pseudomonas tested, P. entomophila secretes a strong diffusible hemolytic activity (see Supplementary Fig. 4 online) that may also be involved in pathogenicity against $D$. melanogaster. We identified three genes unique to $P$. entomophila that may be responsible for this activity (Table 2). The gene encoding PSEEN3925, a putative repeats-in-toxin (RTX) protein, is clustered with genes encoding a type I secretion system. PSEEN0968 and PSEEN3843 are proteins related to outer membrane autotransporters that have been associated with virulence in other bacteria. A number of lipases have also been shown to confer hemolytic activity. The P. entomophila genome encodes four lipases that are absent from P. putida KT2440 and that may contribute to its hemolytic activity (PSEEN709, PSEEN1065, PSEEN2195, PSEEN3432). Interestingly, the gene encoding a lysophospholipase (PSEEN709) is found in a
Figure 2 Comparison of the $P$. entomophila and $P$. putida genomes. (a) Regions of significant sequence identity between the nucleotide sequence of $P$. entomophila (top) and $P$. putida KT2440 (bottom). Colinear regions are connected by red lines and inverted regions by blue lines. The display was generated using Artemis Comparison Toll (freely available at http://www.sanger.ac.uk/Software/ACT/). (b) Specific gene content comparison of the genomes of $P$. entomophila and $P$. putida KT2440. Specific genes of $P$. entomophila $(P e)$ and of $P$. putida KT2440 $(P p)$ with no ortholog in the other species are indicated in blue and green respectively, and are classified according to role categories as described in Figure $\mathbf{1}$. Two genes were considered as orthologs when their products share more than $60 \%$ identity over more than $80 \%$ of their length. Duplicated genes indicated by light colors were detected by using a constraint of 35\% identity over more than $80 \%$ of the length of the protein. Aa, amino acid biosynthesis; $\mathrm{Bc}$, biosynthesis of cofactors, prosthetic groups and carriers; $\mathrm{Ce}$, cell envelope; $\mathrm{Cp}$, cellular processes; $\mathrm{Ci}$, central intermediary metabolism; Dm, DNA metabolism; Em, energy metabolism; Fam, fatty acid and phospholipid metabolism; Me, mobile and extrachromosomal element functions; P, protein synthesis and fate; Pp, purines, pyrimidines, nucleosides and nucleotides; Rf, regulatory functions and signal transduction; Sm, secondary metabolite biosynthesis; T, transcription; Tb, transport and binding proteins; Uf, unknown function and hypothetical proteins.

genomic islet associated with two genes encoding proteins related to insecticidal toxins.

Proteases constitute another important group of extracellular, biologically active substances that are thought to contribute to the virulence of bacterial species. P. entomophila encodes three serine proteases (PSEEN3027, PSEEN3028, PSEEN4433) and an alkaline protease (PSEEN1550) absent from P. putida KT2440. These four genes are located at synteny break points between the genomes of P. entomophila and other Pseudomonas spp. PSEEN1550 is the homo$\log$ of the alkaline protease AprA, which has been shown to be involved in various virulence processes among different species ${ }^{18}$. AprA likely plays a key role in virulence because pathogenicity is affected in mutants defective in PrtR, the predicted transcriptional regulator of aprA (see below).

Pathogenic bacteria rely on a variety of cell surface-associated virulence factors that allow adhesion to the host surface and promote effective colonization. Filamentous hemagglutinin-like adhesins are broadly important virulence factors in both plant and animal pathogens. The genome of $P$. entomophila encodes three proteins (PSEEN0141, PSEEN2177, PSEEN3946) that are predicted to be involved in adhesion and cluster with genes encoding type I or two-partner secretion system proteins (Table 2). We also noticed the presence of two putative autotransporter proteins with a pertactintype adhesion domain.

\section{Toxins against competitors}

In addition to the putative toxins described above that may be crucial for its entomopathogenic properties, P. entomophila carries a number of genes specifying diverse traits that may be required not only for interaction with insects but also for its lifestyle in soil, aquatic or rhizosphere environments (see Supplementary Fig. 5 online).

Fluorescent pseudomonads are characterized by the production of pyoverdines, a diverse class of siderophores containing a chromophore linked to a small peptide of varying length and composition synthesized by nonribosomal peptide synthases ${ }^{19}$. In P. entomophila, the two gene clusters that encode proteins required for pyoverdine biosynthesis and uptake (PSEEN1813-PSEEN1815 and PSEEN3224-3234) present a general organization similar to that found in other fluorescent pseudomonads ${ }^{20}$. We also identified a gene cluster responsible 
Table 2 Gene/gene products potentially involved in P. entomophila-D. melanogaster interaction

Gene/gene producta,b,c Function Ps. ${ }^{\mathrm{d}}$

\begin{tabular}{|c|}
\hline Adhesion \\
\hline PSEEN0141 ${ }^{\mathrm{a}}$ \\
\hline PSEEN2177a \\
\hline PSEEN3946a \\
\hline PSEEN3161 \\
\hline PSEEN4310a \\
\hline Proteases \\
\hline$a p r A^{\mathrm{c}}$ \\
\hline PSEEN3027 ${ }^{b}$ \\
\hline PSEEN3028 ${ }^{\mathrm{b}}$ \\
\hline PSEEN4433a \\
\hline Lipases \\
\hline PSEEN0709 ${ }^{b}$ \\
\hline PSEEN1065 \\
\hline PSEEN2195 \\
\hline PSEEN3432 \\
\hline Toxins \\
\hline$h c n A B C^{c}$ \\
\hline PSEEN0132/3332/3042-5a,b \\
\hline PSEEN2138-56 \\
\hline PSEEN2716-20 \\
\hline PSEEN5524-36 a,b \\
\hline PSEEN0701 ${ }^{\mathrm{a}, \mathrm{b}}$ \\
\hline PSEEN0702 ${ }^{a, b}$ \\
\hline PSEEN1172a \\
\hline PSEEN2485a,c \\
\hline PSEEN2697a,b,c \\
\hline PSEEN2788 \\
\hline PSEEN3326a,b \\
\hline PSEEN3925-9a \\
\hline cellaneous \\
\hline PSEEN0968 a,b \\
\hline PSEEN3843a \\
\hline
\end{tabular}

Noninfectious and nonlethal Tn5 derivatives ${ }^{i}$

\section{$\operatorname{gac} S^{(5)}$}

$\operatorname{gac} A^{(2)}$

bioC $^{(1)}$

PSEEN5207(1)-8(2)

PSEEN4425(2)

Infectious and nonlethal $\operatorname{Tn} 5$ derivatives ${ }^{i}$

prtR $R^{(3)}$

algR(2)

PSEEN0132(3)-3(1)

PSEEN0389(1)
Putative surface adhesion protein

$54 \%$ PP0168

Putative filamentous hemagglutinin

Putative filamentous hemagglutinin

Putative autotransporter, pertactin-like protein

Putative autotransporter, pertactin-like protein

Alkaline metalloprotease

72\% PSPT03332

Putative autotransporter, SSP-h1 serine protease

Putative autotransporter, serine protease

Putative subtilisin-like serine protease

$68 \%$ PSPT0 1650

$64 \%$ PA3535

Absent

Lysophospholipase

Phospholipase C

Triacylglycerol lipase

Lipase class3

$62 \%$ PFL0888

64\% Pf B52 (P21773)

$48 \%$ Pfo0 149
Hydrogen cyanide production

Cluster involved in lipopeptide I biosynthesis

Cluster involved in lipopeptide II biosynthesis

Cluster involved in lipopeptide III biosynthesis

Cluster involved in polyketide biosynthesis

Protein related to TccC-type insecticidal toxin

Protein related to TccC-type insecticidal toxin

Protein related to TcdB-type insecticidal toxin

TccC-type insecticidal toxin

TccC-type insecticidal toxin

TccC-type insecticidal toxin

Putative toxin (cytolethal distending toxin B domains)

Putative RTX toxin and type I secretion system

Putative autotransporter with unknown passenger domain

Putative autotransporter with unknown passenger domain

Absent

53\% PSPTO0714

76\% PA2193 (hcnA)

See text ${ }^{\text {h }}$

Absent

77\% Pfo2266 (2717)

Absent

Absent ${ }^{f}$

Absent ${ }^{\dagger}$

Absent $^{\dagger}$

Absent

Absent

Absent

Absent

Absent

$88 \%$ PP 1650

$98 \%$ PP4099

$86 \%$ PP0365

97\%/82\% PP0283-2

$62 \%$ PFL4631

$74 \%$ PP2889

Transmembrane transcriptional regulator

91\% PP0185

Transcriptional regulator involved in alginate production

NRPS loading protein, CHP (operonic)

Putative chorismate mutase, operonic with $g / n A, n t r B C$
59\%/75\% PSPT05546-7

$44 \%$ PFL0385

aGene products specific to P. entomophila and not found in other Pseudomonas species (constraint of $60 \%$ identity over more than $80 \%$ of the protein length).

bUnusual GC content (differing by more than $1 \mathrm{s.d}$. from the average GC) likely due to recent lateral transfer.

${ }^{\circ} \mathrm{Gene}$ products or predicted domains associated with virulence in other systems.

dSequence identity between the protein encoded by P. entomophila and the best BLAST hit among proteins from other Pseudomonas. PP, P. putida KT2440; PA, P. aeruginosa PA01; PSPTO,

P. syringae pv. tomato DC3000; PFL, P. fluorescens Pf-5; Pfo P. fluorescens PfO-1 and Pf, P. fluorescens.

ePSEENO141 and PPO168 are aligned only on $67 \%$ of PPO168 length.

${ }^{f}<40 \%$ identity.

STrEMBL accession number.

hThis cluster and similarity with that of $P$. fluorescens Pf- 5 are discussed in the Supplementary Figure 5.

iSuperscripted numerals indicate the number of independent $T n 5$ insertions.

iConserved hypothetical protein. 


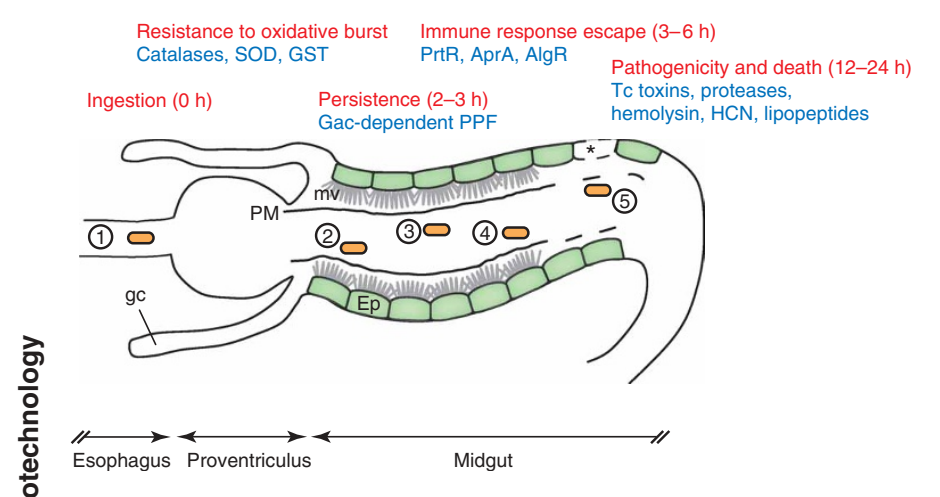

for the synthesis of a siderophore related to acinetobactin and containing a salicylamide moiety ${ }^{21}$ (Supplementary Fig. 5).

Five gene clusters that direct the production of secondary metabolites have been identified (see Supplementary Fig. 5). PSEEN5520PSEEN5522 are responsible for hydrogen cyanide production that is involved in Caenorhabditis elegans killing by $P$. aeruginos $a^{22}$ and in the suppression of soil-borne plant pathogens by certain Pseudomonas species $^{23}$. The genome of $P$. entomophila contains four clusters of genes predicted to encode three different lipopeptides and a polyketide (Table 2 and Supplementary Fig. 5).

Regulation of virulence revealed by a genome-wide mutagenesis To directly identify factors that modulate the interaction between P. entomophila and D. melanogaster, we generated a Tn5-derived library of variants that were individually screened for their infectious and pathogenic properties. Among the 7,500 clones, we isolated 23 mutants whose growth was not affected and that displayed attenuated infectious and/or pathogenic properties (Table 2). Identification of the mini-Tn 5 insertion sites identified directly only a putative lipopeptide as a virulence factor. No other genes predicted to be virulence factors were identified, indicating a likely redundancy. By contrast, a number of insertions affected regulators that likely modulate the expression of such virulence factors. Seven independent insertions inactivated the two-component system GacS/GacA involved in the regulation of various processes, including virulence in different species, and resulted in the inability of these mutants to induce an immune response. P. entomophila gac mutants are defective in secretion of protease and hemolysin (data not shown) and do not persist in the gut of D. melanogaster ${ }^{1}$, indicating the pivotal role of $\mathrm{GacS} / \mathrm{GacA}$ in modulating the entomopathogenic properties of that strain. As observed in other Pseudomonas species ${ }^{23}$, the GacS/GacA twocomponent system probably regulates $P$. entomophila virulence genes at a post-transcriptional level via the two identified small noncoding RsmY and RsmZ RNAs that alleviate post-transcriptional repression by RsmA and RsmE homologs. Three independent insertions in the prtR gene reduce the pathogenic properties of $P$. entomophila but retain the capacity to induce an immune response. In $P$. fluorescens LS107d2 (ref. 24), PrtR and PrtI regulate the transcription of the aprA-inh-aprDEF operon suggesting that P. entomophila relies on AprA protease to fully express its pathogenic properties in D. melanogaster. Two independent insertions that had the same consequences for the interaction with $D$. melanogaster have been found in algR. In $P$. aeruginosa, AlgR regulates a number of processes including fimbrial biogenesis, biofilm formation and cyanide production $^{25,26}$. Altogether, genetic analysis indicates that GacA is a master regulator of the interaction and that PrtR and AlgR regulators, seem to play secondary roles in the infection process.
Figure 3 Steps in the interaction between $P$. entomophila and D. melanogaster. Five different steps are shown: 1 . ingestion of $P$. entomophila through the esophagus; 2 . resistance to oxidative stress in response to a oxidative burst in the gut; 3 . persistence of $P$. entomophila in the gut; 4 . escape from immune response effectors; 5. pathogenicity and lethal outcome of the interaction after important modifications of the midgut physiology including microvilli disruption, cell destruction (indicated by $a^{*}$ ) and in some cases peritrophic matrix disorganization (indicated by a dashed line). Red indicates important steps in the infection process. Blue indicates newly identified proteins that could be involved at these steps in the process. Time scale is indicated in brackets. Ep, epithelial cell; mv, microvilli; PM, peritrophic matrix; gc, gastric cecum.

\section{Metabolism, transport and regulation}

The $P$. entomophila genome encodes most of the central metabolic pathways found in the other Pseudomonas including the pentose phosphate pathway, the Entner-Doudoroff pathway and the tricarboxylic acid cycle. Consistent with Pseudomonas metabolism, P. entomophila has an incomplete Embden-Meyerhof-Parnas pathway owing to the absence of 6-phosphofructokinase, and relies on a complete EntnerDoudoroff route for hexose utilization. The P. entomophila genome harbors several genes that encode hydrolytic activities such as chitinases, lipases and proteases as well as a set of 19 uncharacterized hydrolases, which are potentially involved in the degradation of polymers found in the soil. However, contrary to phytopathogenic strains such as P. syringa $e^{5-7}$, the genome of P. entomophila is devoid of genes encoding enzymes capable of degrading plant cell walls. This is consistent with the observation that this species is not pathogenic for plants (M. Arlat, Institut National de la Recherche Agronomique, Castanet, France, personal communication).

The $P$. entomophila genome also contains determinants for the catabolism of various aromatic compounds (see Supplementary Fig. 6 online) and long-chain carbohydrates. P. entomophila shares several gene clusters with $P$. putida ${ }^{27}$ that are involved in the degradation of various classes of aromatic compounds including benzoate and quinate, 4-hydroxybenzoate, phenylacetaldehyde and phenylalkanoate as well as phenylalanine and tyrosine. The P. entomophila genome contains two additional catabolic gene clusters present in the genome of $P$. aeruginosa PAO1 that encode determinants for the degradation of 3-hydroxybenzoate through gentisate ${ }^{28}$ and for the meta-cleavage of homoprotocatechuate ${ }^{29,30}$.

Consistent with the size of its genome, P. entomophila possesses more than 535 transporter-encoding genes. Remarkably, no genes encoding a type III or type IV secretion system, present in numerous Gram-negative bacterial pathogens ${ }^{31}$, were found in P. entomophila. The high numbers of transcriptional regulators (more than 300) and genes whose products are involved in signal transduction suggests that $P$. entomophila is able to adapt to substantial substrate variations in its habitats.

\section{The soil and entomopathogenic lifestyle of $\boldsymbol{P}$. entomophila}

The metabolic properties of $P$. entomophila predicted from its genome suggest that this strain is a ubiquitous, metabolically versatile bacterium that may colonize diverse habitats including soil, rhizosphere and aquatic systems as shown for $P$. putida KT2440. However, in contrast to $P$. putida, $P$. entomophila contains a number of genes that are predicted, or have been shown, to be important for virulence. The expression of these factors is under the control of the major regulator GacA and presumably allows this strain to exploit new niches and interact with various insects, particularly D. melanogaster (Fig. 3). 
In D. melanogaster, an environment hostile for microbial colonization is maintained in the gut by secretion of antimicrobial factors such as lysozymes ${ }^{32,33}$ and other digestive enzymes. Recently, it has been shown that a unique epithelial oxidative burst limits microbial proliferation in the gut $^{34}$; resistance to oxidative stress might therefore be a prerequisite for $D$. melanogaster gut colonization. The $P$. entomophila genome encodes 40 proteins that are predicted to be involved in resistance to oxidative stress including four catalases, two superoxide dismutases, three hydroperoxide reductases and eleven glutathione- $S$-transferases. It is noteworthy that resistance to oxidative stress is probably not sufficient for colonization as other Pseudomonas species that possess a large repertoire of oxidant detoxifying proteins are not able to persist in the gut of D. melanogaster $^{1}$. This assumption is further reinforced by the observation that $P$. entomophila gacA mutants were not less resistant to peroxide, hypochlorite or paraquat (data not shown). As the P. entomophila- $D$. melanogaster interaction is specific, $P$. entomophila infectivity likely involves the expression of a specific gene enabling this strain to persist in the D. melanogaster gut, as shown for the Erwinia carotovora Evf factor ${ }^{35}$. Because P. entomophila does not contain any evf-related genes, we cannot predict candidates for this putative persistence promoting factor ( $p p f$ in Fig. 3). Nonetheless, this gene is likely regulated by the GacS/GacA two-component system: the gacA::Tn5 or gacS::Tn5 mutants do not persist in the 을 gut and $P$. entomophila cells are infectious only at stationary phase, concomitant with Gac activation of virulence genes (data not shown). It is striking to note that in both $P$. entomophila and E. carotovora ${ }^{35}$, genes required to interact with D. melanogaster are under the control of global regulators, that is, Hor and GacA, respectively, revealing the branching of virulence genes in a complex regulatory network.

Infection of $D$. melanogaster by $P$. entomophila is accompanied by blockage of food-uptake ${ }^{1}$. This phenomenon is also observed in the interaction between Serratia entomophila and the grass grub Costelytra zealandica or between Yersinia pestis and the flea. The processes used to effect food blockage seem to be different in the two systems; Y. pestis (2) relies on phospholipase synthesis and biofilm formation ${ }^{36,37}$ whereas the mechanism used by $S$. entomophila remains unknown ${ }^{38}$. Genes responsible for the anti-feeding determinants of $S$. entomophila have a prophage origin and no related genes were identified in the genome of $P$. entomophila. Since algR mutants still provoke food-uptake blockage, biofilm formation is probably not essential for $D$. melanogaster infection by $P$. entomophila.

The persistence of $P$. entomophila in the larval gut triggers both a local and systemic immune response ${ }^{1}$. The $P$. entomophila level remains high in wild-type larvae, similar to that observed in a relish mutant unable to induce an immune response ${ }^{1}$, suggesting that this strain is able to escape the D. melanogaster immune response. Biofilm formation might protect $P$. entomophila cells from immune effectors or persistence of bacteria might result from the degradation of effectors. The defects observed with prtR mutants indicated that AprA may degrade antimicrobial peptides, as indicated by recent in vivo studies ${ }^{39}$, and consequently disable the immune response.

Twelve hours after D. melanogaster ingests the bacteria, physiological modifications to the fly caused by $P$. entomophila are dramatic and the expression of 205 D. melanogaster genes is modified ${ }^{1}$ (Fig. 3). These changes probably result from the action of virulence factors such as proteases, hemolysins, insecticidal toxin-like proteins, secondary metabolites or hydrogen cyanide. However, lethality starts to be apparent after $16 \mathrm{~h}$, indicating that this late gene expression will have no effect on the fatal outcome of the interaction.

\section{DISCUSSION}

The complete genome sequence of $P$. entomophila provides insight into this organism's entomopathogenic lifestyle. Combined with a genetic approach, it has revealed potential virulence factors along with regulators that modulate their expression. This study also revealed that P. entomophila is the first Pseudomonas strain to be pathogenic in a multicellular organism and at the same time to be devoid of a type III secretion system. Its potential to use various plant-derived compounds including aromatic molecules, and its antibiotic- and oxidative stressresistance capacities suggest that $P$. entomophila is a commensal bacterium. As this strain is not a plant pathogen, it may have potential to control insects. Unexpectedly for an environmental isolate, $P$. entomophila has a genome that contains a limited number of bacteriophages and transposons. This may contribute to its relatively small size compared to other Pseudomonas genomes. Finally, the complete genome sequence of $P$. entomophila provides a framework for further studies to characterize its pathogenic properties and for a host-pathogen system in which both organisms are amenable to genetic and genomic analysis.

\section{METHODS}

Genome sequencing, assembly and annotation. The complete genome sequence of $P$. entomophila L48 was determined using the whole-genome shotgun method $(10 \times$ coverage, using two plasmid libraries and one BAC library to order contigs). Finishing was performed by PCR amplification from contigs extremities. After a first round of annotation, regions of lower quality as well as regions with putative frameshifts were resequenced from PCR amplification of the dubious regions. Using the AMIGene software (annotation of microbial genes) ${ }^{40}$, a total of 5,279 CoDing Sequences were predicted (and assigned a unique identifier prefixed with "PSEEN"), and submitted to automatic functional annotation: exhaustive BLAST searches against the UniProt databank were performed to determine significant homology. Protein motifs and domains were documented using the InterPro databank. In parallel, genes coding for enzymes were classified using the PRIAM software ${ }^{41}$. TMHMM vs2.0 was used to identify transmembrane domains ${ }^{42}$, and SignalP 3.0 was used to predict signal peptide regions ${ }^{43}$. Finally, tRNAs were identified using tRNAscan-SE ${ }^{44}$. Sequence data for comparative analyses were obtained from the NCBI databank (RefSeq section). Putative orthologs and synteny groups (that is, conservation of the chromosomal colocalization between pairs of orthologous genes from different genomes) were computed between P. entomophila and all the other complete genomes as previously described ${ }^{45}$. Manual validation of the automatic annotation was performed using the MaGe (Magnifying Genomes) interface, which allows graphic visualization of the $P$. entomophila annotations enhanced by a synchronized representation of synteny groups in other genomes chosen for comparisons ${ }^{45}$. All the data (that is, syntactic and functional annotations, and results of comparative analysis) were stored in a relational database, called EntomoScope. This database is publicly available via the MaGe interface at http:// www.genoscope.cns.fr/agc/mage/.

Bacterial mutagenesis and screening. Random mutagenesis was performed by biparental mating using $P$. entomophila ${ }^{1}$ and Escherichia coli S17.1- $\lambda$ pir ${ }^{46}$ carrying the pUT- $T n 5-T c$ suicide plasmid as previously described ${ }^{47}$. A total of 7,500 TcR colonies obtained from several independent conjugations were screened individually as previously described ${ }^{35}$. Transconjugants that displayed attenuated virulence were subjected to several secondary screenings by natural infection as previously described ${ }^{1}$. Insertion sites were determined using two different methods. First, genomic DNA was digested by PstI or NotI/PstI and ligated into pUC18 and pBlueScript, respectively. Clones that contained the mini-transposon and its flanking sequences were selected by plating the E. coli BW25142 transformants on tetracycline $(10 \mu \mathrm{g} / \mathrm{ml})$. One flanking region was sequenced from the $T c$ gene using the oligonucleotide (Tc-F) 5'-TCGTCGACA AGCTTCGG-3'. Some insertion sites were determined by reverse PCR method. Genomic DNA was digested by either PstI or EagI, self-ligated and amplified using the oligonucleotides Tc-F and 5'-AGATCTGATCAAGAGACAT-3' 
for PstI-digested DNA or 5'-GGCGGCCCTATACCTTGTCTG-3' (Tet-end) and 5'-CATAATGGGGAAGGCCAT-3' for EagI-digested DNA, respectively. One flanking region was sequenced using the oligonucleotides Tc-F or Tet-end. Insertion sites were confirmed by amplifying the region overlapping the insertion site. Southern blot analysis was carried out to verify that the selected clones only carried a single copy of the transposon.

Accession numbers. The P. entomophila nucleotide sequence and annotation data have been deposited in the EMBL databank under accession number CT573326.

Note: Supplementary information is available on the Nature Biotechnology website.

\section{ACKNOWLEDGMENTS}

This work was supported by CNRS (Programme Séquençage à grande échelle), by IFR 115 and by MRT/ACI IMPBio 2004 'MicroScope.' We thank Célia Floquet and Camille Jourlain for technical assistance, Matthieu Arlat for plant assays and helpful discussions, Alexandra Gruss, Linda Sperling and Sean Kennedy for critical reading of the manuscript, Olivier Espéli for expert annotation. N.V. was supported by a doctoral fellowship from the Association Vaincre la Mucoviscidose and the Association pour la Recherche sur le Cancer

\section{AUTHOR CONTRIBUTIONS}

N.V., V.B., P.W., B.S., J.W., B.L., C.M. and F.B. designed research; N.V., D.V., S.C., Z.R., V.B., C.A., L.C., C.J., A.L., B.V. and F.B. performed research; N.V., D.V., S.C., V.B., C.A., C.M. and F.B. contributed new reagents/analytic tools; N.V., D.V., S.C., Z.R., V.B., C.A., L.C., C.J., A.L., B.V., B.L., C.M. and F.B. analyzed data; and N.V. and F.B. wrote the paper.

\section{COMPETING INTERESTS STATEMENT}

The authors declare that they have no competing financial interests.

Published online at http://www.nature.com/naturebiotechnology/

Reprints and permissions information is available online at http://npg.nature.com/ reprintsandpermissions/

1. Vodovar, N. et al. Drosophila host defense after oral infection by an entomopathogenic Pseudomonas species. Proc. Natl. Acad. Sci. USA 102, 11414-11419 (2005).

2. Waterfield, N.R., Wren, B.W. \& ffrench-Constant, R.H. Invertebrates as a source of emerging human pathogens. Nat. Rev. Microbiol. 2, 833-841 (2004).

3. Chattopadhyay, A., Bhatnagar, N.B. \& Bhatnagar, R. Bacterial insecticidal toxins. Crit. Rev. Microbiol. 30, 33-54 (2004).

4. Stover, C.K. et al. Complete genome sequence of Pseudomonas aeruginosa PAO1, an opportunistic pathogen. Nature 406, 959-964 (2000).

Buell, C.R. et al. The complete genome sequence of the Arabidopsis and tomato pathogen Pseudomonas syringae pv. tomato DC3000. Proc. Natl. Acad. Sci. USA 100, 10181-10186 (2003)

6. Feil, H. et al. Comparison of the complete genome sequences of Pseudomonas syringae pv. syringae B728a and pv. tomato DC3000. Proc. Natl. Acad. Sci. USA 102, 1106411069 (2005)

7. Joardar, V. et al. Whole-genome sequence analysis of Pseudomonas syringae pv. phaseolicola 1448 A reveals divergence among pathovars in genes involved in virulence and transposition. J. Bacteriol. 187, 6488-6498 (2005).

8. Paulsen, I.T. et al. Complete genome sequence of the plant commensal Pseudomonas fluorescens Pf-5. Nat. Biotechnol. 23, 873-878 (2005).

9. Nelson, K.E. et al. Complete genome sequence and comparative analysis of the metabolically versatile Pseudomonas putida KT2440. Environ. Microbiol. 4, 799808 (2002).

10. Weinel, C., Nelson, K.E. \& Tummler, B. Global features of the Pseudomonas putida KT2440 genome sequence. Environ. Microbiol. 4, 809-818 (2002).

11. Eisen, J.A., Heidelberg, J.F., White, O. \& Salzberg, S.L. Evidence for symmetric chromosomal inversions around the replication origin in bacteria. Genome Biol $\mathbf{1}$ RESEARCH0011 (2000).

12. Aranda-Olmedo, I., Tobes, R., Manzanera, M., Ramos, J.L. \& Marques, S. Speciesspecific repetitive extragenic palindromic (REP) sequences in Pseudomonas putida. Nucleic Acids Res. 30, 1826-1833 (2002).

13. Waterfield, N.R., Bowen, D.J., Fetherston, J.D., Perry, R.D. \& ffrench-Constant, R.H. The tc genes of Photorhabdus: a growing family. Trends Microbiol 9, 185-191 (2001).

14. Bowen, D. et al. Insecticidal toxins from the bacterium Photorhabdus luminescens. Science 280, 2129-2132 (1998).

15. Joo Lee, P. et al. Cloning and heterologous expression of a novel insecticidal gene (tccC1) from Xenorhabdus nematophilus strain. Biochem. Biophys. Res. Commun. 319, 1110-1116 (2004).
16. Waterfield, N., Hares, M., Yang, G., Dowling, A. \& ffrench-Constant, R. Potentiation and cellular phenotypes of the insecticidal Toxin complexes of Photorhabdus bacteria. Cell Microbiol. 7, 373-382 (2005).

17. Wilson, M., McNab, R. \& Henderson, B.. Bacterial Disease Mechanisms (Cambridge University Press, Cambridge, UK, 2002).

18. Miyoshi, S. \& Shinoda, S. Microbial metalloproteases and pathogenesis. Microbes Infect. 2, 91-98 (2000).

19. Meyer, J.M. Pyoverdines: pigments, siderophores and potential taxonomic markers of fluorescent Pseudomonas species. Arch. Microbiol. 174, 135-142 (2000).

20. Ravel, J. \& Cornelis, P. Genomics of pyoverdine-mediated iron uptake in pseudomonads. Trends Microbiol. 11, 195-200 (2003).

21. Mercado-Blanco, J. et al. Analysis of the pmsCEAB gene cluster involved in biosynthesis of salicylic acid and the siderophore pseudomonine in the biocontrol strain Pseudomonas fluorescens WCS374. J. Bacteriol. 183, 1909-1920 (2001).

22. Gallagher, L.A. \& Manoil, C. Pseudomonas aeruginosa PAO1 kills Caenorhabditis elegans by cyanide poisoning. J. Bacteriol. 183, 6207-6214 (2001).

23. Haas, D. \& Defago, G. Biological control of soil-borne pathogens by fluorescent pseudomonads. Nat. Rev. Microbiol. 3, 307-319 (2005).

24. Burger, M., Woods, R.G., McCarthy, C. \& Beacham, I.R. Temperature regulation of protease in Pseudomonas fluorescens LS107d2 by an ECF sigma factor and a transmembrane activator. Microbiology 146, 3149-3155 (2000).

25. Lizewski, S.E. et al. Identification of AlgR-regulated genes in Pseudomonas aeruginosa by use of microarray analysis. J. Bacteriol. 186, 5672-5684 (2004).

26. Whitchurch, C.B. et al. Phosphorylation of the Pseudomonas aeruginosa response regulator AlgR is essential for type IV fimbria-mediated twitching motility. J. Bacteriol. $184,4544-4554$ (2002)

27. Jimenez, J.I., Minambres, B., Garcia, J.L. \& Diaz, E. Genomic analysis of the aromatic catabolic pathways from Pseudomonas putida KT2440. Environ. Microbiol. 4, 824841 (2002).

28. Liu, D.Q., Liu, H., Gao, X.L., Leak, D.J. \& Zhou, N.Y. Arg169 is essential for catalytic activity of 3-hydroxybenzoate 6-hydroxylase from Klebsiella pneumoniae M5a1. Microbiol. Res. 160, 53-59 (2005)

29. Prieto, M.A., Diaz, E. \& Garcia, J.L. Molecular characterization of the 4-hydroxyphenylacetate catabolic pathway of Escherichia coli W: engineering a mobile aromatic degradative cluster. J. Bacteriol. 178, 111-120 (1996).

30. Thotsaporn, K., Sucharitakul, J., Wongratana, J., Suadee, C. \& Chaiyen, P. Cloning and expression of p-hydroxyphenylacetate 3-hydroxylase from Acinetobacter baumannii: evidence of the divergence of enzymes in the class of two-protein component aromatic hydroxylases. Biochim. Biophys. Acta 1680, 60-66 (2004).

31. Hueck, C.J. Type III protein secretion systems in bacterial pathogens of animals and plants. Microbiol. Mol. Biol. Rev. 62, 379-433 (1998).

32. Hultmark, D. Insect lysozymes. EXS 75, 87-102 (1996).

33. Regel, R., Matioli, S.R. \& Terra, W.R. Molecular adaptation of Drosophila melanogaster lysozymes to a digestive function. Insect Biochem. Mol. Biol. 28, 309-319 (1998).

34. Ha, E.M. et al. An antioxidant system required for host protection against gut infection in Drosophila. Dev. Cell 8, 125-132 (2005).

35. Basset, A., Tzou, P., Lemaitre, B. \& Boccard, F. A single gene that promotes interaction of a phytopathogenic bacterium with its insect vector, Drosophila melanogaster. EMBO Rep. 4, 205-209 (2003).

36. Hinnebusch, B.J. et al. Role of Yersinia murine toxin in survival of Yersinia pestis in the midgut of the flea vector. Science 296, 733-735 (2002).

37. Darby, C., Ananth, S.L., Tan, L. \& Hinnebusch, B.J. Identification of gmhA, a Yersinia pestis gene required for flea blockage, by using a Caenorhabditis elegans biofilm system. Infect. Immun. 73, 7236-7242 (2005).

38. Hurst, M.R., Glare, T.R. \& Jackson, T.A. Cloning Serratia entomophila antifeeding genes-a putative defective prophage active against the grass grub Costelytra zealandica. J. Bacteriol. 186, 5116-5128 (2004).

39. Liehl, P., Blight, M., Vodovar, N., Boccard, F. \& Lemaitre, B. Prevalence of local immune response against oral infection in a Drosophila/Pseudomonas infection model. PLoS Pathog., in the press.

40. Bocs, S., Cruveiller, S., Vallenet, D., Nuel, G. \& Medigue, C. AMIGene: Annotation of MIcrobial Genes. Nucleic Acids Res. 31, 3723-3726 (2003).

41. Claudel-Renard, C., Chevalet, C., Faraut, T. \& Kahn, D. Enzyme-specific profiles for genome annotation: PRIAM. Nucleic Acids Res. 31, 6633-6639 (2003).

42. Krogh, A., Larsson, B., von Heijne, G. \& Sonnhammer, E.L. Predicting transmembrane protein topology with a hidden Markov model: application to complete genomes. J. Mol. Biol. 305, 567-580 (2001).

43. Bendtsen, J.D., Nielsen, H., von Heijne, G. \& Brunak, S. Improved prediction of signal peptides: SignalP 3.0. J. Mol. Biol. 340, 783-795 (2004).

44. Lowe, T.M. \& Eddy, S.R. tRNAscan-SE: a program for improved detection of transfer RNA genes in genomic sequence. Nucleic Acids Res. 25, 955-964 (1997).

45. Vallenet, D. et al. MaGe: a microbial genome annotation system supported by synteny results. Nucleic Acids Res. 34, 53-65 (2006).

46. Miller, V.L. \& Mekalanos, J.J. A novel suicide vector and its use in construction of insertion mutations: osmoregulation of outer membrane proteins and virulence determinants in Vibrio cholerae requires toxR. J. Bacteriol. 170, 2575-2583 (1988).

47. de Lorenzo, V., Herrero, M., Jakubzik, U. \& Timmis, K.N. Mini-Tn5 transposon derivatives for insertion mutagenesis, promoter probing, and chromosomal insertion of cloned DNA in gram-negative eubacteria. J. Bacteriol. 172, 6568-6572 (1990). 